\title{
On the Jones Index Values for Conformal Subnets
}

\author{
Sebastiano Carpi* \\ Dipartimento di Scienze, Università di Chieti-Pescara "G. d'Annunzio" \\ Viale Pindaro, 42, I-65127 Pescara, Italy \\ E-mail: carpi@sci.unich.it \\ YASUYUKI KAWAHIGASHI ${ }^{\dagger}$ \\ Department of Mathematical Sciences \\ University of Tokyo, Komaba, Tokyo, 153-8914, Japan \\ E-mail: yasuyuki@ms.u-tokyo.ac.jp \\ RoBerto LONGO* \\ Dipartimento di Matematica, Università di Roma "Tor Vergata", \\ Via della Ricerca Scientifica, 1, I-00133 Roma, Italy \\ E-mail: longo@mat.uniroma2.it
}

February 18, 2010

\begin{abstract}
We consider the smallest values taken by the Jones index for an inclusion of local conformal nets of von Neumann algebras on $S^{1}$ and show that these values are quite more restricted than for an arbitrary inclusion of factors. Below 4, the only non-integer admissible value is $4 \cos ^{2} \pi / 10$, which is known to be attained by a certain coset model. Then no index value is possible in the interval between 4 and $3+\sqrt{3}$. The proof of this result based on $\alpha$-induction arguments. In the case of values below 4 we also give a second proof of the result. In the course of the latter proof we classify all possible unitary braiding symmetries on the $A D E$ tensor categories, namely the ones associated with the even vertices of the $A_{n}, D_{2 n}, E_{6}, E_{8}$ Dynkin diagrams.
\end{abstract}

\footnotetext{
*Supported in part by PRIN-MIUR, GNAMPA-INDAM and EU network "Noncommutative Geometry" MRTN-CT-2006-0031962

†Supported in part by the Grants-in-Aid for Scientific Research, JSPS.
} 
Supported in part by the ERC Advanced Grant 227458 OACFT "Operator Algebras and Conformal Field Theory"

\section{Introduction}

A celebrated theorem by V.F.R. Jones [15] states that for an inclusion of factors $N \subset M$ the possible values for the index are $[M: N]=4 \cos ^{2} \pi / n, n=3,4, \ldots$ or $[M: N] \geq 4$. All the values $4 \cos ^{2} \pi / n$ in the discrete series are realized and the in this case the inclusion is automatically irreducible, i.e. $N^{\prime} \cap M=\mathbb{C}$. It is also rather easy to construct a reducible inclusion of factors with any index value larger or equal to 4 . All the values above 4 are realized for non-hyperfinite irreducible subfactors as in [22]. Whether every value larger than 4 can be realized as the index of an irreducible inclusion of hyperfinite factors is still an open problem, we mention that deep progresses have been performed in the classification of finite depth subfactors, in particular by Popa, Haagerup and Asaeda, see [23, 13, 1, 2].

In this paper we begin to study the possible values of the index for an inclusion $\mathcal{A} \subset \mathcal{B}$ of local conformal subnet on $S^{1}$. Indeed only Möbius covariance will enter in our discussion. Note that here, if the index of $\mathcal{A} \subset \mathcal{B}$ is finite, then the inclusion $\mathcal{A} \subset \mathcal{B}$ is automatically irreducible, i.e. $\mathcal{A}(I)^{\prime} \cap \mathcal{B}(I)=\mathbb{C}$ for any interval $I$ of $S^{1}$, see [8, Corollary 2.7].

We shall see that the possible index values for $\mathcal{A} \subset \mathcal{B}$ below $3+\sqrt{3}$ are $1,2,3$, $4 \cos ^{2} \pi / 10$ and 4 . So only one non-integer value in the Jones discrete series appears here: the value $4 \cos ^{2} \pi / 10$. An inclusion of conformal net with index $4 \cos ^{2} \pi / 10$ has been considered by Rehren [24]. This is pointed out in [9] where one can also find further partial results related to this paper.

The integer index values are easily realized as orbifold, indeed if $\mathcal{A}$ is the fixedpoint net of $\mathcal{B}$ under a finite gauge symmetry group $G$ then the index of $\mathcal{A} \subset \mathcal{B}$ is the order of $G$, so it suffices to consider for example the cyclic orbifold of $\mathcal{C} \otimes \cdots \otimes \mathcal{C}$ for any conformal net $\mathcal{C}$.

One might compare our result with the restriction on the index values for a sector of a local conformal net [18, 25]. Also in this case there are definitely more index value gaps than in the subfactor case (in particular only four index values between 4 and 6 are possible), yet there is no obvious direct link between the two cases.

We shall give two proofs of our results. The first one is based on $\alpha$-induction arguments, has the advantage of being shorter and works for all the index values below $3+\sqrt{3}$. The second proof is, in a sense, more conceptual but it works only for the index values below 4. It can be viewed as a result for braided tensor categories. The basic idea is the following. Since local extensions of a conformal net correspond to local $Q$-systems in the tensor category of DHR sectors of the net [19, 20], our problem is of purely tensor categorical nature and it is sufficient to consider any local conformal net whose representation tensor category contains our given braided tensor category. We basically shall use $S U(2)_{n}$-models to this end.

Indeed we shall need to classify all possible unitary braiding symmetries for the 
tensor categories associated with the even vertices of a Dynkin diagram of type $A_{n}$, $D_{2 n}, E_{6}$ and $E_{8}$ (the Dynkin diagrams associated with a subfactor with index less than 4). This result, which has its own interest, might be known to some extent; it can be derived by means of Izumi quantum double analysis [14], but is apparently not available in the literature, cf. however [17].

\section{$2 \alpha$-induction and restriction on the index values}

We consider possible index values of inclusions of local conformal nets. Let $\mathcal{A}(I) \subset$ $\mathcal{B}(I)$ be an inclusion of local conformal nets with finite index. (See [11] for basic notions and results of the Jones index theory [15].) Here the Möbius covariance for $\mathcal{A}, \mathcal{B}$ is enough and we do not need $\operatorname{Diff}\left(S^{1}\right)$-covariance here. We use the machinery of $\alpha$-induction, which has been introduced in [20] and studied in [26, 4]. (Strong additivity of a net is sometimes assumed, but we do not need this assumption.)

Consider the principal and dual principal graphs of the subfactor $\mathcal{A}(I) \subset \mathcal{B}(I)$ for a fixed interval $I \subset S^{1}$. We label $\mathcal{A}-\mathcal{A}, \mathcal{B}-\mathcal{A}$, and $\mathcal{B}-\mathcal{B}$ sectors by symbols $\lambda, b, \tau$, respectively. Note that the $\mathcal{A}-\mathcal{A}$ sectors arising from this subfactor are DHR sectors of the local net $\mathcal{A}$. We denote the inclusion map of this subfactor by $\iota$ and regard it as a $\mathcal{B}-\mathcal{A}$ sector. The dual canonical endomorphism $\theta$ is given by $\bar{\iota} \iota$ and it is regarded as an $\mathcal{A}-\mathcal{A}$ sector.

Fix an irreducible $\mathcal{A}-\mathcal{A}$ sector $\lambda$ (until the end of this paragraph). Let $\iota \lambda=\sum_{b} n_{b} b$ be the irreducible decomposition of the $\mathcal{B}-\mathcal{A}$ sector $\iota \lambda$, where $n_{b}$ is a nonnegative integer representing the multiplicity. We also let $\alpha_{\lambda}=\sum_{\tau} m_{\tau} \tau$ and $b \bar{\iota}=\sum_{\tau} k_{b, \tau} \tau$ be the irreducible decompositions, where $b$ is an arbitrary $\mathcal{B}-\mathcal{A}$ sector. Note that we have

$$
n_{b}=\langle\iota \lambda, b\rangle=\left\langle\alpha_{\lambda} \iota, b\right\rangle=\left\langle\alpha_{\lambda}, b \bar{\iota}\right\rangle=\sum_{\tau} k_{b, \tau} m_{\tau}
$$

By locality, the $\alpha$-induction has the following property.

$$
\langle\iota \lambda, \iota \lambda\rangle=\left\langle\alpha_{\lambda}, \alpha_{\lambda}\right\rangle
$$

as in [26, Proof of Theorem 3.3]. (Also see [4, Part I, Theorem 3.9].) This implies the following.

$$
\sum_{b}\left(\sum_{\tau} k_{b, \tau} m_{\tau}\right)^{2}=\sum_{\tau} m_{\tau}^{2}
$$

By expanding the left hand side, we have

$$
\sum_{b, \tau, \tau^{\prime}} k_{b, \tau} k_{b, \tau^{\prime}} m_{\tau} m_{\tau^{\prime}}=\sum_{\tau} m_{\tau}^{2}
$$

which implies

$$
\sum_{\tau}\left(\sum_{b} k_{b, \tau}^{2}\right) m_{\tau}^{2} \leq \sum_{\tau} m_{\tau}^{2}
$$


For any $\tau$ with $m_{\tau}>0$, there exists $b$ with $b \prec \tau \iota$, and for this $b$, we have $k_{b, \tau}=$ $\langle\tau, b \bar{\iota}\rangle=\langle\tau \iota, b\rangle>0$. Then the above inequality (10) implies that there exists only one $b$ with $k_{b, \tau}>0$, and for this $b$, we have $k_{b, \tau}=1$. This shows that if we have $m_{\tau}>0$, then there exists a unique irreducible $b$ with $\tau \iota=b$.

Now choose an arbitrary irreducible $\mathcal{B}-\mathcal{A}$ sector $b$ appearing in the dual principal graph. Then there exists $\lambda$ with $b \prec \iota \lambda=\alpha_{\lambda} \iota$. Since

$$
1 \leq\langle\iota \lambda, b\rangle=\left\langle\alpha_{\lambda} \iota, b\right\rangle=\left\langle\alpha_{\lambda}, b \bar{\iota}\right\rangle
$$

there exists an irreducible $\mathcal{B}$ - $\mathcal{B}$ sector $\tau$ with $\tau \prec \alpha_{\lambda}$ and $\tau \prec b \bar{\iota}$. By the arguments in the previous paragraph, we have $\tau \iota=b$. This has proved the following.

Theorem 2.1. In the above setting, for any odd vertex $b$ of the dual principal graph of the subfactor $\mathcal{A}(I) \subset \mathcal{B}(I)$, we have an even vertex $\tau$ of the dual principal graph that is connected only to $b$.

This gives the following corollary immediately, since if we take the odd vertex having distance 3 from the initial vertex $*$ as $b$ in the above theorem, then the property of the theorem is violated.

Corollary 2.2. Consider a bipartite graph $G$ with the initial vertex *. Suppose $G$ has a vertex with valency larger than 2 and let $d$ be the distance from $*$ to the nearest vertex with valency larger than 2. If $d>3$, then the graph $G$ is not the dual principal graph of a subfactor arising from an irreducible local extension of a local conformal net.

If the Jones index is less than 4, the principal graph must be one of the $A-D_{2 n}-E_{6,8}$ diagrams. Also in this case, the principal graph and the dual principal graph are the same. Only $A_{2}, A_{3}, A_{5}, D_{4}$ and $D_{6}$ satisfy the condition in Theorem 2.1. Note that for any finite groups $H \subset G$, we can realize $G$ as a subgroup of the automorphism group of a local conformal net $\mathcal{M}$ through permutations of the tensor components. (That is, we embed $G$ into some symmetric group $S_{n}$ of order $n$ and realized $\mathcal{M}$ as the $n$th tensor power of some local conformal net. Then $G$ acts on $\mathcal{M}$ through permutations.) Then we have an inclusion of local conformal net $\mathcal{A}=\mathcal{M}^{G} \subset \mathcal{M}^{H}=$ $\mathcal{B}$. The principal graphs $A_{2}, A_{3}, A_{5}, D_{4}$ can be realized with the choices of $(G, H)=$ $(\{e\},\{e\}),\left(S_{2},\{e\}\right),\left(S_{3}, S_{2}\right),(\mathbb{Z} / 3 \mathbb{Z},\{e\})$, where $e$ denotes the identity element in a group.

We know that the coset construction for the diagonal embedding $S U(2)_{3} \subset S U(2)_{2} \otimes$ $S U(2)_{1}$ gives the Virasoro net $\operatorname{Vir}_{7 / 10}$. (See [16, Section 3] based on [27].) Then the embedding $S U(2)_{3} \otimes \operatorname{Vir}_{7 / 10} \subset S U(2)_{2} \otimes S U(2)_{1}$ has principal graph $D_{6}$ as noted in [24, (5.6)]. Thus the principal graph $D_{6}$, with the index value $4 \cos ^{2} \pi / 10$, is realized for an inclusion of local conformal nets.

Next we consider the case beyond index 4. Haagerup [13] has considered possible principal graphs for the index range $(4,3+\sqrt{3})$ and shown that such graphs are severely restricted. Today it has been determined that three of his pairs of finite graphs are realized and the others are not. (See [1, 2, 3] and references there.) It is 
easy to see that none of Haagerup's graphs survive the restrictions given in Corollary 2.2. (Note that Corollary 2.2 applies also to an infinite graph and excludes $A_{\infty}$, which is in the list of Haagerup [13].)

At the index value $3+\sqrt{3}$, we have an inclusion of local conformal nets arising from conformal embedding $S U(2)_{10} \subset S O(5)_{1}$, which was studied in [26]. (Note that this

subfactor is isomorphic to the well-known GHJ-subfactor [12, Section 4.5] arising from $E_{6}$, up to tensoring the common injective type $\mathrm{III}_{1}$ factor, as shown in [6, Proposition A.3].)

By these considerations, we obtain the following theorem.

Theorem 2.3. The smallest five values of the Jones indices of inclusions of local conformal nets are $1,2,3,4 \cos ^{2} \pi / 10,4$ and $3+\sqrt{3}$.

\section{Classification of braidings}

We now present a different method to determine which index values below 4 are possible for inclusions of local conformal nets. Note that if an inclusion $\mathcal{A}(I) \subset \mathcal{B}(I)$ of local conformal nets has index below 4 , the system of $\mathcal{A}(I)-\mathcal{A}(I)$ bimodules given by the even vertices of the principal graph has to have a braiding, since this gives a full subcategory of the DHR-category of the representations of the local conformal net $\mathcal{A}(I)$. The principal graph in this case must be one of the $A_{n}, D_{2 n}, E_{6}, E_{8}$ diagrams, so we will classify all possible braidings on the even vertices of these graphs in this section.

Ocneanu realized that one can classify braidings by studying the "quantum double" system [21], and we follow the formulation of Izumi in [14], based on the Longo-Rehren subfactor [20]. (We actually follow a formulation in [7], where a definition dual to that in [14] is used.) Consider a system $\Delta$ of irreducible endomorphisms with finite indices for a type III factor $M$. If we have a braiding $\varepsilon(\lambda, \mu)$ on the system $\Delta$, it also gives a half-braiding naturally, so we have a system $\{(\lambda, \varepsilon(\lambda, \cdot)) \mid \lambda \in \Delta\}$ of irreducible objects, which gives a subcategory of the quantum double category, and this system has the same fusion rules as $\Delta$ by [14] Conversely, if we have a subcategory of the quantum double category generated by a system $\left\{\left(\lambda, \mathcal{E}_{\lambda}(\cdot)\right) \mid \lambda \in \Delta\right\}$ of irreducible objects, where $\mathcal{E}_{\lambda}(\cdot)$ is a half-braiding, and this system has the same fusion rules as $\Delta$, then it gives a braiding on $\Delta$ by [14, Theorem 4.6 (ii), (iii)]. Hence, for computing the number of braidings, up to equivalence, it is enough to count the number of embeddings of $\Delta$ to $\left\{\left(\lambda, \mathcal{E}_{\lambda}(\cdot)\right) \mid \lambda \in \Delta\right\}$, with the same fusion rules in the quantum double category. The quantum double category for the $A_{n}, D_{2 n}, E_{6}, E_{8}$ subfactors have been computed in [21], [10], [14], [7, so we simply count the number of such embeddings below.

We deal with five cases separately, and the first three cases are further divided into subcases.

Case I: $A_{2 n}$.

Subcase Ia: $A_{2}$. 
In this case, the subfactor has index 1 , and we have only one even vertex. So the number of braidings is trivially 1 .

Subcase Ib: $A_{2 n}$ with $n>1$. It is known that the braiding arising from $S U(2)_{2 n-1}$ restricted on the even vertices is nondegenerate. (See [21], [10], [14].) So the quantum double system is simply given by doubling as in [14, Corollary 7.2], and the irreducible objects are labeled with pairs $(j, k)$ with $j, k \in\{0,2,4, \ldots, 2 n-2\}$ where the irreducible objects of the $S U(2)_{2 n-1}$ category are labeled with $0,1,2, \ldots, 2 n-1$ as usual. Then the odd vertices of the dual principal graph of the Longo-Rehren subfactor $M \otimes M^{\text {opp }} \subset R$ are labeled with $l \in\{0,2,4, \ldots, 2 n-2\}$, and the number of the edges connecting $(j, k)$ and $l$ is given by the structure constant $N_{j k}^{l}$ by [14, Section 4]. Then it is easy to see that only possible embeddings are given by $j \mapsto(j, 0)$ and $j \mapsto(0, j)$. So the number of the braidings is 2 .

Case II: $A_{2 n+1}$. We again label the irreducible objects of the $S U(2)_{2 n}$ category with $0,1,2, \ldots, 2 n$. The braiding arising from $S U(2)_{2 n}$ restricted on the even vertices is degenerate and the quantum double system is explicitly described, as in [10], [14, Section 7].

Subcase IIa: $A_{3}$. The even vertices of $A_{3}$ are given by the group $\mathbb{Z} / 2 \mathbb{Z}$. We draw the induction-restriction graph for the quantum double system for the Longo-Rehren subfactor $M \otimes M^{\text {opp }} \subset R$. Then it is easy to see that we have 2 embeddings.

Subcase IIb: $A_{5}$. It was found by Ocneanu that the number of braidings is 3 . We present arguments here for the sake of completeness as follows.

The dual principal graph of the Longo-Rehren subfactor $M \otimes M^{\text {opp }} \subset R$ for this case is given in [10, Figure 21]. It is easy to see that the vertex labeled with 2 can be mapped to 3 irreducible sectors labeled with $02,20,22_{-}$and each indeed gives an embedding in the above sense. Thus the number of the braidings is 3 . The restriction of the original braiding on $S U(2)_{4}$ gives 2 of them, and the other comes from realization of the even vertices of $A_{5}$ from the dual of $S_{3}$, which gives a completely degenerate braiding, where all monodromy operators are trivial.

Subcase IIc: $A_{2 n+1}$ with $n>2$. We label the irreducible objects of the quantum double system with $(j, k)$ with $j, k \in\{0,1,2, \ldots, 2 n\}$ satisfying $j+k \in 2 \mathbb{Z}$ and $(j, k) \neq(n, n)$ with identification $(j, k)=(2 n-j, 2 n-k)$, and $(n, n)_{+},(n, n)_{-}$as in [10, Section 4], [14, Section 7]. The statistical dimension of $(j, k)$ is

$$
\sin ((j+1) \pi /(2 n+2)) \sin ((k+1) \pi /(2 n+2)) / \sin ^{2}(\pi /(2 n+2))
$$

and that of $(n, n)_{ \pm}$is $1 / 2 \sin ^{2}(\pi /(2 n+2))$, and now $n>2$, so only $(0,2),(0,2 n-2)$, $(2,0),(2 n-2,0)$ have the same statistical dimensions as the irreducible object 2 of $S U(2)_{2 n}$. From this, we see that the only possible embeddings are given by $j \mapsto(j, 0)$ and $j \mapsto(0, j)$. So the number of braidings is 2 .

Case III: $D_{2 n}$. Note that the even vertices of the $D_{2 n}$ diagram are realized as the DHR sectors of the local extensions of the $S U(2)_{4 n-4}$-nets with index 2 as in 44, Part II]. This gives at least 2 non-degenerate embeddings.

Subcase IIIa: $D_{4}$. The even vertices of $D_{4}$ are given by the group $\mathbb{Z} / 3 \mathbb{Z}$. By similar arguments to the case $A_{3}$, we see we have 3 embeddings. Two of them come 
from the local extension of the $S U(2)_{4}$-net, and the other is a degenerate one coming from $\mathbb{Z} / 3 \mathbb{Z} \cong \widehat{\mathbb{Z} / 3 \mathbb{Z}}$.

Subcase IIIb: $D_{6}$. The even vertices of $D_{6}$ are realized as the irreducible DHR sectors of a local extension of $S U(2)_{8}$ of index 2 with the dual canonical endomorphism $\theta=0 \oplus 8$. The $\alpha$-inductions of 0 and 2 are irreducible, and that of 4 has an irreducible decomposition into two pieces, so we label them with $0,2,4_{+}, 4_{-}$, respectively. Then the fusion rules are commutative and non-trivial ones are as follows.

$$
\begin{aligned}
2 \cdot 2 & =0+2+4_{+}+4_{-}, \\
2 \cdot 4_{+} & =2+4_{+}, \\
2 \cdot 4_{-} & =2+4_{-}, \\
4_{ \pm} \cdot 4_{ \pm} & =0+4_{ \pm}, \\
4_{ \pm} \cdot 4_{\mp} & =2 .
\end{aligned}
$$

Then the even vertices of the dual principal graph of the Longo-Rehren subfactor $M \otimes M^{\text {opp }} \subset R$ are labeled with pairs $(j, k)$ with $j, k \in\left\{0,2,4_{+}, 4_{-}\right\}$. The odd vertices of the dual principal graph are labeled with $l \in\left\{0,2,4_{+}, 4_{-}\right\}$and the number of edges connecting $(j, k)$ and $l$ is given by the structure constant $N_{j k}^{l}$. We now count the number of embeddings of the system $\left\{0,2,4_{+}, 4_{-}\right\}$into the quantum double system. From the above fusion rules, it is easy to see that 2 has to be mapped to one of $(0,2),(2,0),\left(4_{+}, 4_{-}\right),\left(4_{-}, 4_{+}\right)$. Then it is also easy to see that all of these give embeddings, so the number of the braidings is 4 .

These four braidings are interpreted as follows. Since the braiding of the even vertices of $A_{4}$ is nondegenerate as see above, its quantum double system is simply a self-doubling. That is, if we label the even vertices of $A_{4}$ with $1, \sigma$, where 1 is the identity sector, then the even vertices of $D_{6}$ are labeled as $1 \otimes 1,1 \otimes \sigma, \sigma \otimes 1$, and $\sigma \otimes \sigma$. Since the system $\{1, \sigma\}$ has braidings $\varepsilon^{ \pm}$arising from $S U(2)_{3}$, the system $\{1 \otimes 1,1 \otimes \sigma, \sigma \otimes 1, \sigma \otimes \sigma\}$ has four braidings $\varepsilon^{+} \otimes \varepsilon^{+}, \varepsilon^{+} \otimes \varepsilon^{-}, \varepsilon^{-} \otimes \varepsilon^{+}$and $\varepsilon^{-} \otimes \varepsilon^{-}$. The above consideration show that these four exhaust all the possibilities.

Subcase IIIc: $D_{2 n}$ with $n>3$. We label the even vertices of $D_{2 n}$ as $0,2,4, \ldots, 2 n-$ $2_{+}, 2 n-2_{-}$based on the $\alpha$-induction as above. The irreducible objects of the quantum double system are labeled with pairs of these again. If $n>5$, then the statistical dimension of 2 is the smallest among $2,4, \ldots, 2 n-2_{+}, 2 n-2_{-}$and this shows that only possible embeddings arise from $2 \mapsto(0,2)$ and $2 \mapsto(2,0)$. For the cases $n=4,5$, the fusion rules directly show that these are also only possibilities. They give 2 embeddings, and the number of the braidings is again 2 .

Case IV: $E_{6}$. It was found by Ocneanu that the system arising from the even vertices of $E_{6}$ has no braiding. This seems to be well-known to experts, but we present arguments here as follows for the sake of completeness.

The dual principal graph of the Longo-Rehren subfactor arising from the even vertices of $E_{6}$ is given in [7, Figure 1]. With the labeling used in this Figure, an embedding has to map 2 to one of $(2,0),(8,0),(1,1),(5,1)_{1},(5,1)_{2}$, but none of these give the correct $E_{6}$ fusion rules. For example, we have $(2,0) \cdot(2,0)=(0,0) \oplus$ $(2,0) \oplus(4,0)$. This shows we have no embeddings. 


\begin{tabular}{|c|c|c|c|c|c|c|c|}
\hline$A_{2}$ & $A_{5}$ & other $A_{n}$ & $D_{4}$ & $D_{6}$ & other $D_{2 n}$ & $E_{6}$ & $E_{8}$ \\
\hline 1 & 3 & 2 & 3 & 4 & 2 & 0 & 0 \\
\hline
\end{tabular}

Table 1: The numbers of braidings

Case V: $E_{8}$. This is very similar to Case IV and the same remark applies. We now use [7, Figure 2]. Any embedding has to map 2 to $(2,0)$, but this does not give the correct $E_{8}$ fusion rules, so we have no embeddings.

Combining all the above, we obtain the following theorem.

Theorem 3.1. For each system of bimodules given by the even vertices of one of the $A_{n}-D_{2 n}-E_{6,8}$ diagrams, the number of braidings is given as in Table 1 .

\section{Another proof on the restriction on the index values}

We now give an alternative proof of the restriction on the index values for inclusions of local conformal nets based on the results in the previous section.

Suppose that one of the $A_{n}-D_{2 n}-E_{6,8}$ diagrams is realized as a principal graph for an inclusion $\mathcal{A}(I) \subset \mathcal{B}(I)$ of local conformal nets. The even vertices of the principal graph must give DHR-sectors of the net $\mathcal{A}$, so they must have a braiding. By Theorem 3.1, the graphs $E_{6}$ and $E_{8}$ are excluded.

Suppose the graph is $A_{n}, n \neq 3,5$. By Theorem 3.1, all the braidings arise from $S U(2)_{n-1}$, and $\mathcal{A}(I) \subset \mathcal{B}(I)$ is a local extension, so we can copy the $Q$-system [19], 20] of $\mathcal{A}(I)$ so that the net $S U(2)_{n-1}$ also has a local extension with the dual canonical endomorphism $\theta=0 \oplus 2$. The classification table in [16, Theorem 2.4] shows that this is impossible. So only $A_{3}, A_{5}$ remain, and we already know they are indeed realized as in Section 2 .

Now suppose the graph is $D_{2 n}, n \neq 2,3$. Again by Theorem 3.1, all the braidings arise from the local extension of $S U(2)_{4 n-4}$ with index 2, and $\mathcal{A}(I) \subset \mathcal{B}(I)$ is a local extension, so we can copy the $Q$-system of $\mathcal{A}(I)$ so that the local extension of $S U(2)_{4 n-4}$ also has a further local extension. This is a local extension of $S U(2)_{4 n-4}$ with the dual canonical endomorphism $\theta=0 \oplus 2 \oplus(4 n-6) \oplus(4 n-4)$. The classification table in [16, Theorem 2.4] shows that this is again impossible. So only $D_{4}, D_{6}$ remain, and we already know they are indeed realized as in Section 2, Note that in the case of $D_{6}$, the braiding arising from the local extension of $S U(2)_{8}$ with index 2 does not allow a further local extension, but the representation category of $S U(2)_{3} \otimes \operatorname{Vir}_{7 / 10}$ contains a subcategory corresponding to the even vertices of $D_{6}$, and the braiding arising from this local conformal net does allow a local extension to $S U(2)_{2} \otimes S U(2)_{1}$.

We thus obtain the following theorem again.

Theorem 4.1. The smallest four values of the Jones indices of inclusions of local conformal nets are $1,2,3,4 \cos ^{2} \pi / 10$ and 4 . 
Remark 4.2. In the above arguments on the restriction of possible index values, all we need is a local $Q$-system arising from a unitary braided tensor category. So we have the same conclusion for index values for such $Q$-systems.

Remark 4.3. We have a similar problem for index values also for inclusions of 2dimensional conformal nets. The same arguments for the restriction works. The integer index values are again all possible with orbifold nets.

We see that the remaining value $4 \cos ^{2} \pi / 10$ is also possible as follows. Consider the $S U(2)_{3}$-net and label the irreducible DHR sectors as $\lambda_{0}, \lambda_{1}, \lambda_{2}, \lambda_{3}$, where $\lambda_{0}$ is the vacuum representation. Then the Longo-Rehren inclusion is given by a $Q$-system

$\bigoplus_{j=0,1,2,3} \lambda_{j} \otimes \lambda_{j}^{\text {opp }}$, but the endomorphism $\bigoplus_{j=0,2} \lambda_{j} \otimes \lambda_{j}^{\text {opp }}$ also gives a $Q$-system with localiy, hence a local extension. This gives the index value $4 \cos ^{2} \pi / 10$. So in this setting, we have the same result as in Theorem 4.1.

Acknowledgmentes. This work has been partly done during visits of S.C. and R.L. at the University of Tokyo and of Y.K. at the University of Rome "Tor Vergata"; we thank both institutions for the warm hospitality.

\section{References}

[1] M. Asaeda \& U. Haagerup, Exotic subfactors of finite depth with Jones indices $(5+\sqrt{13}) / 2$ and $(5+\sqrt{17}) / 2$, Commun. Math. Phys. 202 (1999) 1-63.

[2] M. Asaeda \& S. Yasuda, On Haagerup's list of potential principal graphs of subfactors, Commun. Math. Phys. 286 (2009) 1141-1157.

[3] S. Bigelow, S. Morrison, E. Peters \& N. Snyder, Constructing the extended Haagerup planar algebra, arXiv:0909.4099.

[4] J. Böckenhauer \& D. E. Evans, Modular invariants, graphs and $\alpha$-induction for nets of subfactors I, Commun. Math. Phys. 197 (1998) 361-386. II 200 (1999) 57-103. III 205 (1999) 183-228.

[5] J. Böckenhauer, D. E. Evans \& Y. Kawahigashi, On $\alpha$-induction, chiral projectors and modular invariants for subfactors, Commun. Math. Phys. 208 (1999) 429487.

[6] J. Böckenhauer, D. E. Evans \& Y. Kawahigashi, Chiral structure of modular invariants for subfactors, Commun. Math. Phys. 210 (2000) 733-784.

[7] J. Böckenhauer, D. E. Evans \& Y. Kawahigashi, Longo-Rehren subfactors arising from $\alpha$-induction, Publ. RIMS Kyoto Univ. 37 (2001) 1-35.

[8] C. D'Antoni, R. Longo \& F. Rădulescu, Conformal nets, maximal temperature and models from free probability, J. Operator Theory 45 (2001) 195-208. 
[9] A. Degan, "On the range of Jones index for inclusions of local nets of von Neumann algebras on the circle", Tesi di Dottorato, Università di Chieti-Pescara "G. d'Annunzio" 2009.

[10] D. E. Evans, Y. Kawahigashi, Orbifold subfactors from Hecke algebras II Quantum doubles and braiding_-, Commun. Math. Phys. 196 (1998) 331-361

[11] D. E. Evans \& Y. Kawahigashi, "Quantum symmetries on operator algebras", Oxford University Press, 1998.

[12] F. Goodman, P. de la Harpe \& V. F. R. Jones, "Coxeter Graphs and Towers of Algebras" MSRI Publications, Springer, Berlin-Heidelberg-New York, 14, 1989.

[13] U. Haagerup, Principal graphs of subfactors in the index range $4<3+\sqrt{2}$, in Subfactors - Proceedings of the Taniguchi Symposium, Katata -, (ed. H. Araki, et al.), World Scientific (1994), 1-38.

[14] M. Izumi, The structure of sectors associated with the Longo-Rehren inclusions I. General theory, Commun. Math. Phys. 213 (2000) 127-179.

[15] V. F. R. Jones, Index for subfactors, Invent. Math. 72 (1983) 1-25.

[16] Y. Kawahigashi \& R. Longo, Classification of local conformal nets. Case $c<1$, Ann. of Math. 160 (2004) 493-522.

[17] D. Kazhdan \& H. Wenzl, Reconstructing monoidal categories. In I. M. Gelfand Seminar, volume 16 of Adv. Soviet Math., pages 111-136. Amer. Math. Soc., Providence, RI, 1993.

[18] R. Longo, Minimal index and braided subfactors, J. Funct. Anal. 109 (1992), 98-112.

[19] R. Longo, A duality for Hopf algebras and for subfactors, Commun. Math. Phys. 159 (1994) 133-150.

[20] R. Longo \& K.-H. Rehren, Nets of subfactors, Rev. Math. Phys. 7 (1995) 567-597.

[21] A. Ocneanu, Chirality for operator algebras (Notes recorded by Y. Kawahigashi), in Subfactors, World Scientific, (1994) 39-63

[22] S. Popa, Markov traces on universal Jones algebras and subfactors of finite index, Invent. Math. 111 (1993), 375-405.

[23] S. Popa, "Classification of Subfactors and Their Endomorphisms", CBMS Regional Conference Series in Mathematics 1995.

[24] K.-H. Rehren, Subfactors and coset models, in Generalized symmetries in physics (Clausthal, 1993), World Scientific, (1994) 338-356. 
[25] K.-H. Rehren, On the range of the index of subfactors, J. Funct. Anal. 134 (1995) 183-193

[26] F. Xu, New braided endomorphisms from conformal inclusions, Commun. Math. Phys. 192 (1998) 347-403.

[27] F. Xu, Algebraic coset conformal field theories I, Commun. Math. Phys. 211 (2000) $1-44$. 